\title{
Using molecular dynamics simulation to study the polarization response of the liquid water interface to surface charge heterogeneity
}

\author{
Sucheol Shin ${ }^{\mathrm{a}, \mathrm{b}}$ and Adam P. Willard ${ }^{\mathrm{a}, 1}$

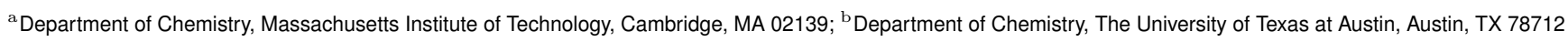 \\ This manuscript was compiled on September 16, 2021
}

The hydration shells of proteins mediate interactions, such as small molecule binding, that are vital to their biological function or in some cases their dysfunction. However, even when the structure of a protein is known, the properties of its hydration environment cannot be easily predicted due to the complex interplay between protein surface heterogeneity and the collective fluctuations of water's hydrogen bonding network. This manuscript presents a theoretical study of the influence of surface charge heterogeneity on the polarization response of the liquid water interface. We introduce a new computational method for analyzing simulation data that is capable of quantifying water's nonlinear polarization response and determining the effective surface charge distribution of hydrated surfaces over atomistic length scales. When applied to a protein, this method is capable of revealing new insight into the influence of conformational dynamics on hydration structure, as we highlight by illustrating how salt-bridge formation enhances the polarization of the local hydration shell. To illustrate the utility of this method, we present the results of molecular dynamics simulations of liquid water in contact with a heterogeneous model surface and the CheY protein.

The external surfaces of proteins (and other large biomolecules) are chemically and topographically heterogeneous. When a protein is solvated, certain signatures of this heterogeneity are evident in the properties of the surrounding solvent. Understanding how these solvation signatures emerge and what information they convey about protein surface composition is important due to their potential role in facilitating selectivity in small molecule binding or driving recognition in the nascent stages of protein-protein interactions (1-7). The protein surface properties that may be reflected in these signatures, such as charge, hydrophobicity, and local curvature, have differing influences on local water structure and dynamics (8-12). These different influences are mixed and diluted by the correlated fluctuations of water's interfacial hydrogen bonding network, making their combined effects hard to disentangle or predict. Theory and molecular simulation can be applied to better understand the collective molecular interactions that determine the solvation properties of proteins and other complex surfaces.

A significant component of the protein solvation signature originates from electrostatic interactions $(1,5)$. The charged and polar residues that give rise to these interactions produce a three-dimensional electric field profile that extends into and polarizes the surrounding aqueous environment. Surface charge heterogeneity is reflected in

14 this electric field profile and thereby also in the molecular structure of the liquid water interface. Here, we aim to

\footnotetext{
${ }^{1}$ To whom correspondence should be addressed. E-mail: awillard@mit.edu
} 
determine how effectively the fluctuating molecular structure of the adjacent water interface communicates the charge distribution of a surface. We consider the effects of electric field on the orientational statistics of interfacial water molecules and demonstrate how these statistics can be analyzed to quantify water's polarization response to surface charge heterogeneity. We find that the local polarization response of the water interface is significantly altered by molecular effects that arise due to surface-bound waters. We demonstrate that these molecular effects are sensitive to protein dynamics, highlighting the importance of considering conformational fluctuations when studying hydration properties.

Many previous studies have been aimed at understanding the microscopic properties of water at charged and polar surfaces. These properties can be probed with surface sensitive spectroscopies, such as sum-frequency generation spectroscopy $(13-15)$ and terahertz absorption spectroscopy $(16,17)$, or with dynamic nuclear polarization NMR $(8,18,19)$. However, ability to experimentally resolve site-specific details of the solvation environment under room temperature conditions is extremely limited. What experiments have revealed is that strong polar surface-water interactions can significantly modify the hydrogen bonding structure of the liquid water interface and reduce the mobility of interfacial water molecules (20-22). These observations are consistent with the results of simulation studies using both classical $(23,24)$ and first-principles $(25,26)$ molecular dynamics (MD) simulation, as well as the predictions of continuum $(27,28)$ and coarse-grained modeling $(29-31)$. While it is clear that charged and polar surfaces have a significant influence on the properties of their aqueous environments, many of the specific details about water's interfacial polarization response remain unknown.

The polarization response of the liquid water interface involves the realignment of water molecules that are interconnected through strong directional hydrogen bonding interactions. The consequences of these interconnections are not captured by continuum models, such as those based on Poisson-Boltzmann or dielectric continuum theory (32-34), nor are the effects of thermal fluctuations, which introduce significant variation over sub-ns timescales. To study the role that these effects play in protein hydration (and the processes that initiate protein interactions), we utilize all-atom MD, which explicitly describes the collective molecular interactions that govern the aqueous hydrogen bonding network and its response to external electric fields. We demonstrate that molecular-scale details of surface charge can be accurately inferred from only about 20 ps of sampled orientational configurations of interfacial water molecules. However, to make this inference it is necessary to account for both the inherent non-linearity of water's polarization response, arising from collective hydrogen bonding interactions, and the excluded volume and Coulombic forces arising from surface-bound molecules. We present a computational method that accounts for these effects and show how it can be applied to monitor the influence of protein dynamics on local solvent polarization.

In the following section, we consider the influence of a generic uniformly charged surface on the orientational distributions of interfacial water molecules. We highlight that these distributions are not related by simple linear response for biologically relevant surface charge densities. We present a method for analyzing water's interfacial polarization that accounts for these nonlinearities and demonstrate how the method can be used to infer a surface's charge distribution. This method is summarized in the subsequent section and described in more detail in the Methods section. By applying this method to a model protein system, we identify how the making and breaking of salt bridges leads to an impulsive change in water's local polarization profile. 


\section{Results and Discussion}

Characterizing polarization response from depth-resolved statistics of interfacial molecular structure. The molecular structure and dynamics of liquid water reflect the strong tendency of molecules to engage in hydrogen bonding. This tendency couples the orientations of adjacent molecules in a way that can influence their response to externally applied electric fields. At an interface, this response is further complicated by the depth-dependent anisotropy of the hydrogen bond network. All-atom MD simulation is an efficient tool for modeling this anisotropy and determining its influence on water's polarization response.

To characterize this influence, we perform simulations of neat liquid water in contact with a model surface that is divided into two oppositely charged regions of tunable surface charge density, $\pm\left|\sigma_{\mathrm{s}}\right|$ (Fig. 1A; see Methods section for more detailed description). We analyze these simulations by determining the depth and orientation of each water molecule relative to the plane of the interface. Following Refs. 35 and 9, we specify these quantities in terms of $\vec{\kappa}=\left(\cos \theta_{1}, \cos \theta_{2}, a\right)$, where $a$ denotes the distance of the water molecule from the nearest point of the instantaneous liquid water interface, and $\theta_{1}$ and $\theta_{2}$ are the angles made between each $\mathrm{OH}$ bond vector and the local surface normal. The conditional probability distribution, $P\left(\vec{\kappa} \mid \sigma_{\mathrm{s}}\right)$, encodes the polarization state of a given interface, and the polarization response can thus be quantified by analyzing how this distribution changes when $\sigma_{\mathrm{s}}$ is varied.

Figure 1A illustrates the sensitivity of interfacial molecular structure to surface charge over a range of positive and negative surfaces. For ease of visualization, we have projected out one of the angular components of $P\left(\vec{k} \mid \sigma_{\mathrm{s}}\right)$ and independently normalized the distribution at each value of $a$ to yield the marginal distributions, $P\left(\cos \theta_{1} \mid a, \sigma_{\mathrm{s}}\right)$. We observe significant changes in molecular structure with changing surface charge that extend over the first $\sim 1 \mathrm{~nm}$ of the interface. The structure in these distributions reflects the distorted interfacial hydrogen bonding network $(31,36,37)$, and its response to the presence of a uniform electric field. We observe a shift in the peak value of $\cos \theta_{1}$ for surface molecules (i.e., with $a \leq 3 \AA$ ) associated with the alignment of water dipoles with the field, i.e., with hydrogens pointed toward or away from the negative or positive surface, respectively. The tilt of subsurface molecules (i.e., with $a \geq 3 \AA$ ) with the electric field of the interface is also evident in these distributions.

The molecular polarization response of the interface can be quantified by comparing the details of $P\left(\vec{\kappa} \mid \sigma_{\mathrm{s}}\right)$ generated at differing values of $\sigma_{\mathrm{s}}$. If this response is linear, then these distributions can be related by a simple Boltzmann reweighting that accounts for the coupling of a given $\vec{\kappa}$ to the external electric field. We evaluate the linearity in the molecular polarization response by assuming that this coupling is given by,

$$
\epsilon=-\vec{\mu} \cdot \vec{E},
$$

where $\epsilon$ is the energy of the dipole of a water molecule, $\vec{\mu}$, in a field $\vec{E}$. The depth-dependent distribution of water dipoles,

$$
P\left(\vec{\mu} \mid a^{\prime}, \sigma_{\mathrm{s}}\right) \propto \int \delta\left(\vec{\mu}_{\kappa}-\vec{\mu}\right) \delta\left(a-a^{\prime}\right) P\left(\vec{\kappa} \mid \sigma_{\mathrm{s}}\right) d \vec{\kappa},
$$

where $\delta(x)=1$ when $x=0$ or otherwise equals 0 , and $\vec{\mu}_{\kappa}$ is the value of the dipole vector given by $\vec{\kappa}$, can be predicted from linear response (LR) as,

$$
P_{\mathrm{LR}}\left(\vec{\mu} \mid a, \sigma_{\mathrm{s}}\right) \propto P\left(\vec{\mu} \mid a, \sigma_{s}=0\right) e^{-\beta \epsilon},
$$



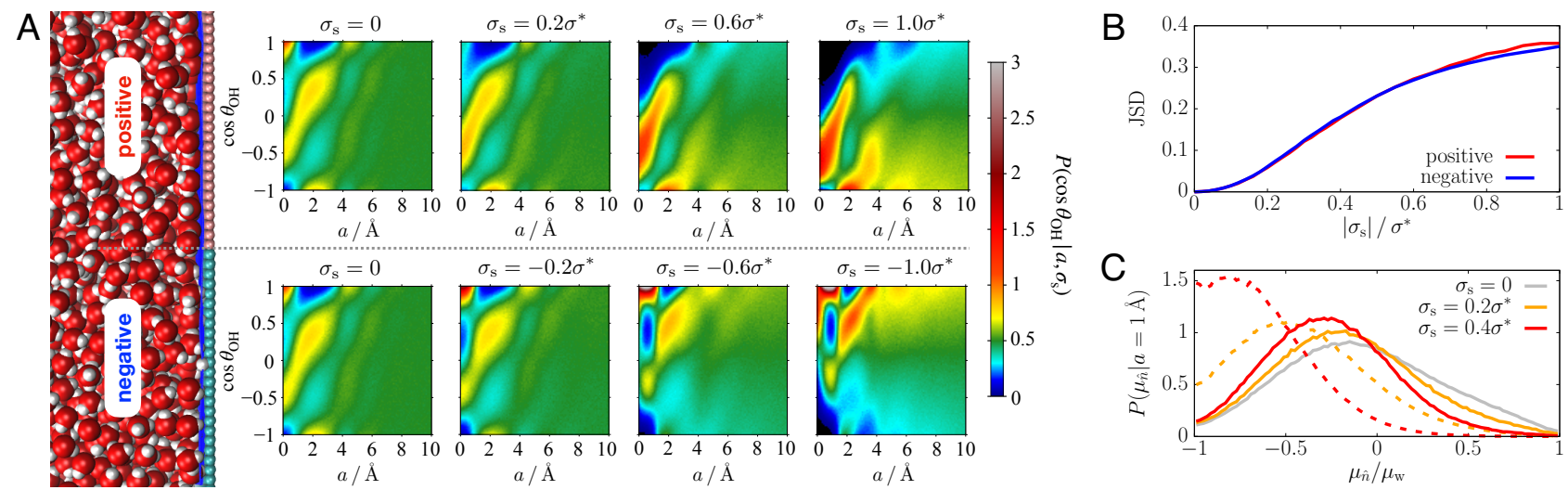

Fig. 1. Effect of surface charge density on the orientational distribution of interfacial water molecules. (A) The left-hand panel contains a simulation snapshot of the charged surface-water interface, where positive and negative surface particles are depicted as pink and cyan spheres, respectively, and the instantaneous liquid water interface is shown in blue. The right-hand panels contain plots of the depth-dependent orientational distribution, $P\left(\cos \theta_{\mathrm{OH}} \mid a, \sigma_{\mathrm{s}}\right)$, for interfacial water molecules at the surfaces with different charge densities, $\sigma_{\mathrm{s}}$, where $\sigma^{*}=0.85 e / \mathrm{nm}^{2}$. The top and bottom panels show the plots for the surfaces with positive and negative charges, respectively. (B) Plots of JSD [Eq. 4], which compare the distribution of interfacial molecular polarization to the linear response (LR) prediction, are given as a function of $\sigma_{\mathrm{s}}$, where the red and blue curves are for $\sigma_{\mathrm{s}}>0$ and $\sigma_{\mathrm{s}}<0$ respectively. (c) Comparison of the distribution of the molecular polarization in the surface normal direction, $\mu_{\hat{n}}$, at $a=1 \AA$ between the simulation (solid line) and LR prediction (dashed line), where $\mu_{\mathrm{w}}$ is the dipole moment of a water molecule and the distributions for $\sigma_{\mathrm{s}}=0,0.2 \sigma^{*}$, and $0.4 \sigma^{*}$ are shown in gray, orange, and red colors, respectively.

where $\epsilon$ in this expression uses the field, $\vec{E}$, associated with the surface charge $\sigma_{\mathrm{s}}$. More computational details about the LR estimation are provided in the SI Appendix.

We quantify the accuracy of the LR assumption by computing the Jensen-Shannon divergence,

$$
\mathrm{JSD}=\frac{1}{2}\left[\bar{D}_{\mathrm{KL}}\left(P\left(\vec{\mu} \mid a, \sigma_{\mathrm{s}}\right)|| P_{\mathrm{M}}\right)+\bar{D}_{\mathrm{KL}}\left(P_{\mathrm{LR}}\left(\vec{\mu} \mid a, \sigma_{\mathrm{s}}\right)|| P_{\mathrm{M}}\right)\right],
$$

where $P_{\mathrm{M}}=P\left(\vec{\mu} \mid a, \sigma_{\mathrm{s}}\right)+P_{\mathrm{LR}}\left(\vec{\mu} \mid a, \sigma_{\mathrm{s}}\right)$ and $\bar{D}_{\mathrm{KL}}$ is the depth-averaged Kullback-Leibler divergence (see the SI appendix for more details). This quantity is a metric of the overlap between the LR prediction and the distribution $P\left(\vec{\mu} \mid a, \sigma_{\mathrm{s}}\right)$ sampled directly from MD simulations. Unlike the KL divergence, JSD is symmetric and has an upper bound $\left(0 \leq \mathrm{JSD} \leq 1\right.$, if $\log _{2}$ is used in evaluating $\left.\bar{D}_{\mathrm{KL}}\right)$. Essentially, JSD $=0$ if $P\left(\vec{\mu} \mid a, \sigma_{\mathrm{s}}\right)$ and $P_{\mathrm{LR}}\left(\vec{\mu} \mid a, \sigma_{\mathrm{s}}\right)$ are identical and JSD $=1$ if the distributions have no overlap. For scale, two Gaussian distributions with identical widths and means separated by their standard deviation yield JSD $\approx 0.16$. The value of JSD computed across a range of different surface charge density is plotted in Fig. 1B. We observe that JSD increases rapidly for $\left|\sigma_{\mathrm{s}}\right| \lesssim 0.4 \sigma^{*}$ while the increase rate gets smaller for $\left|\sigma_{\mathrm{s}}\right|>0.4 \sigma^{*}$. Figure 1C illustrates how the distribution of interfacial polarization differs between the simulation and LR estimate at a given interfacial depth for $\sigma_{\mathrm{s}}=0.2 \sigma^{*}$ and $0.4 \sigma^{*}$. In each case, the molecular dipole distribution shift is less than expected based on the LR estimate. The deviation of $P\left(\vec{\mu} \mid a, \sigma_{\mathrm{s}}\right)$ from the LR estimate indicates that the reorientation of water molecules are significantly affected by the constraints imposed by the interfacial hydrogen bonding network.

Inferring local surface charge from water's interfacial molecular structure. The signatures of water's nonlinear response to surface charge are contained within the conditional probability distribution, $P\left(\vec{\kappa} \mid \sigma_{\mathrm{s}}\right)$. If these distributions are known, then they provide a basis for inferring surface charge from the configurational statistics of interfacial water molecules. To make this inference, we follow a similar procedure to that of Ref. 9, in which local surface hydrophobicity 
is inferred by comparing sampled water configurations to a pre-tabulated distribution, $P(\vec{\kappa} \mid$ phob $)$, which describes water's response to a purely hydrophobic surface. In this subsection, we briefly summarize an approach that adapts this formalism to the problem of determining local surface charge.

To infer surface charge, we employ a strategy based on maximum likelihood estimation (MLE). Specifically, we sample a set of water configurations adjacent to a surface and compare those configurations to $P\left(\vec{\kappa} \mid \sigma_{\mathrm{s}}\right)$ by computing the likelihood that those configurations would be spontaneously sampled at a surface with charge density $\sigma_{\mathrm{s}}$. We define the local surface charge estimate, i.e., the apparent surface charge density $\sigma_{\mathrm{MLE}}$, as the value of $\sigma_{\mathrm{s}}$ with the maximum likelihood. By basing the estimate of surface charge on pre-sampled distributions, i.e., $P\left(\vec{\kappa} \mid \sigma_{\mathrm{s}}\right)$, we implicitly correct for water's underlying nonlinear response. The specific details of our method are described in Methods section.

\section{Evaluating how surface charge heterogeneity is reflected in water's interfacial molecular structure. Many hydrated} surfaces, including those of proteins, have surface charge heterogeneity over molecular length scales. To assess how heterogeneity on these length scales affects the polarization response of the adjacent liquid water interface, we study a tunable model surface in contact with a slab of liquid water. Specifically, our model surface comprises an immobilized snapshot of bulk liquid water molecules at equilibrium as described by the SPC/E force field at $T=300 \mathrm{~K}$ and near conditions of liquid-vapor phase coexistence. The exposed face of this rigid water model surface includes all molecules whose oxygens reside on one side of a plane drawn through a configuration of a periodically replicated slab of the bulk liquid. The model surface thus includes a disordered distribution of surface charge, as given by the partial charges of the immobilized water molecules, that we tune through a uniform scaling by parameter $\alpha$, such that $\alpha=0$ corresponds to an uncharged (hydrophobic) surface and $\alpha=1$ corresponds to the surface with the specific charge amplitudes of liquid water. More details about the model surface are contained within the SI Appendix for preparing this model surface and simulating its aqueous interfacial system.

To study the influence of the heterogeneous surface on water's interfacial polarization, we carry out simulations of a slab of liquid water in contact with model surfaces with a variety of different values of $\alpha$. For each value of $\alpha$, we generate a spatially resolved interfacial polarization map by sampling configurations of interfacial water molecules located over specific points along the surface and using those sampled configurations to compute the corresponding value of $\sigma_{\mathrm{MLE}}$. Figure 2 contains the results of these calculations for the model surface with several different values of $\alpha$. We find that the specific pattern of the actual surface charge distribution, $\sigma_{\mathrm{s}}$ (Fig. 2A; see SI Appendix for the details about estimating $\sigma_{\mathrm{s}}$ ), is accurately reflected in the polarization response of water when $\alpha$ is small, e.g., $\alpha \lesssim 0.4$ (Fig. 2B). However, when $\alpha$ is larger, the map of $\sigma_{\mathrm{MLE}}$ deviates from that of the underlying surface charge (Fig. 2C), indicating the emergence of lateral correlations in water's interfacial polarization response.

To quantify the correspondence between $\sigma_{\mathrm{MLE}}$ and $\sigma_{\mathrm{s}}$, we compare values generated at different points along the model surface. Figure 2D contains a scatter plot of all possible surface points that illustrates this correspondence. We observe much better agreement (i.e. narrower spread) between $\sigma_{\mathrm{MLE}}$ and $\sigma_{\mathrm{s}}$ when $\alpha=0.4\left(R^{2}=0.86\right)$ than when $\alpha=1.0\left(R^{2}=0.65\right)$, where only the qualitative features of charge heterogeneity are reflected in water's interfacial polarization response. We further quantify the correspondence between $\sigma_{\mathrm{MLE}}$ and $\sigma_{\mathrm{s}}$ by computing the mean unsigned 
error (MUE),

$$
\mathrm{MUE}=\frac{1}{N_{\mathrm{surf}}} \sum_{i=1}^{N_{\mathrm{surf}}}\left|\sigma_{\mathrm{MLE}, i}-\sigma_{\mathrm{s}, i}\right|,
$$

where the summation is taken over all $N_{\text {surf }}$ points sampled along the surface. As Fig. 2E illustrates, we observe a significant increase in the difference between $\sigma_{\mathrm{MLE}}$ and $\sigma_{\mathrm{s}}$ for $\alpha \gtrsim 0.4$. This increase signals the onset of non-ideal response, whereby the local polarization of the interface is significantly influenced by lateral molecular correlations. As the more discrete structure of the data plotted in Fig. 2C suggests, these lateral correlations arise when surface sites manage to pin individual water molecules. We provide a quantitative evidence for this interpretation in SI Appendix, Fig. S5 by tracking the number of interfacial water molecules and its fluctuations at a given location on the surface.
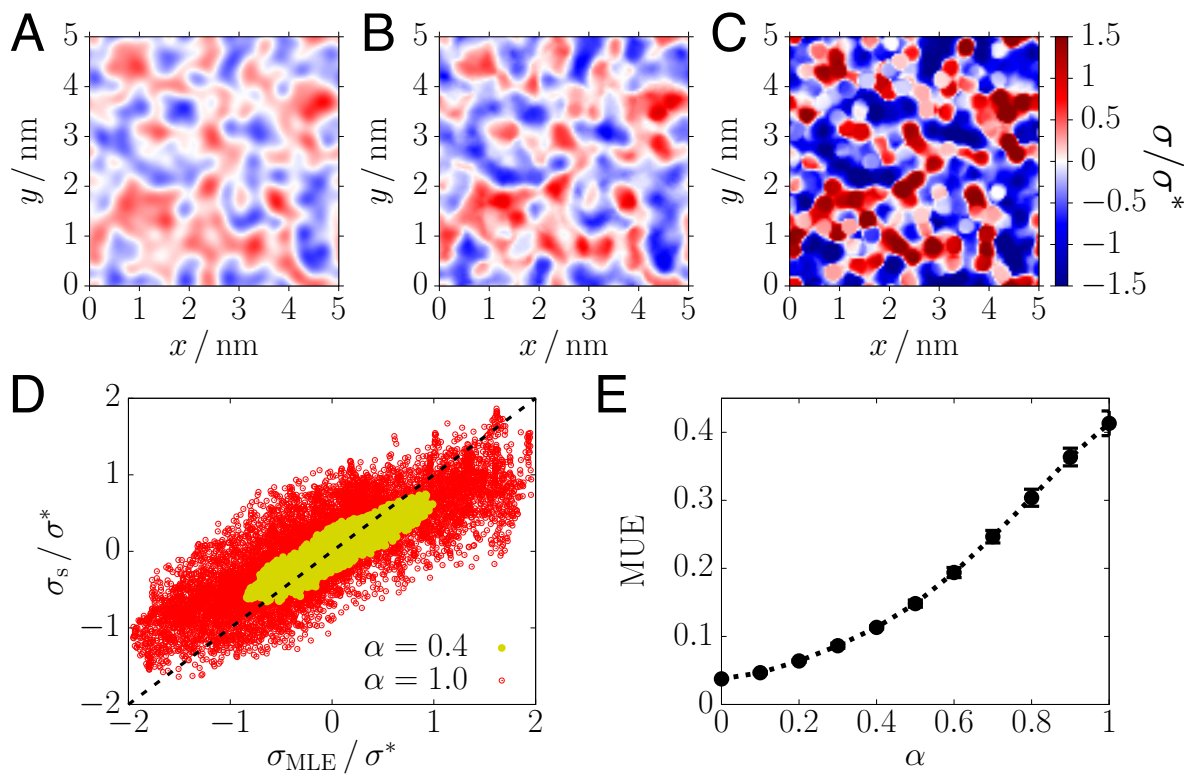

Fig. 2. Surface charge heterogeneity is reflected in water's interfacial molecular structure. (A) A spatial map of the heterogeneous surface charge density, $\sigma_{\mathrm{s}}$, indicated by shading, for $\alpha=0.4$. (B-C) A spatial map of the inferred surface charge density, $\sigma_{\mathrm{MLE}}$, indicated by shading, for $\alpha=0.4$ and $\alpha=1.0$, respectively. Panels (A-C) share the same color scale. (D) A scatter plot illustrating the correlation between the actual $\left(\sigma_{\mathrm{S}}\right)$ and inferred $\left(\sigma_{\mathrm{MLE}}\right)$ values of surface charge density computed at points along the surface with $\alpha=0.4$ (yellow points) and $\alpha=1.0$ (red points). Dashed line represents the perfect correlation, $\sigma_{\mathrm{s}}=\sigma_{\mathrm{MLE}}$. (E) Plot of the $\alpha$ dependence of the mean unsigned error [Eq. 5]. The dotted line is a guide to the eye.

Role of Water-Water Interactions in the Nonlinear Response of Interfacial Water. When strong surface-water interactions cause water molecules to be fully or partially immobilized at specific surface sites, those bound water molecules augment the effective surface charge distribution. In a sense, bound water molecules act as a component of the surface itself. By accounting for the electrostatic influence of surface-bound water molecules, the interfacial polarization response can be more accurately predicted at larger values of $\alpha$.

To account for the electrostatic influence of other water molecules, we evaluate the electric field at the center of each water molecule, due to the surrounding solvent. Then, we project this field perpendicular to the interface and use Gauss's law to infer an effective local surface charge density, $\sigma_{\text {liq }}$ (see SI Appendix for more details). Using this effective surface charge, we define a total effective surface charge density,

$$
\sigma_{\mathrm{tot}}=\sigma_{\mathrm{s}}+\sigma_{\mathrm{liq}},
$$


that combines the influence of the local surface and its impact on the surrounding liquid. The results of this decomposition are presented in Fig. 3 .

Figure 3A contains plots of $\sigma_{\text {liq }}$ for various values of $\alpha$. We observe that $\sigma_{\text {liq }}$ highlights the positions and local electrostatic influence of pinned water molecules (see also SI Appendix, Fig. S6B). As illustrated in Fig. 3B, the predicted surface charge, $\sigma_{\mathrm{MLE}}$, agrees better with the total effective surface charge including $\sigma_{\text {liq }}$ than $\sigma_{\mathrm{s}}$ alone, with mean errors of $0.31 \pm 0.01$ and $0.41 \pm 0.02$ for $\alpha=1$, respectively (see SI appendix, Fig. S6C for the comparison of errors in the full range of $\alpha$.) These results thus highlight the role of water-water interactions in the non-ideal response of $\sigma_{\mathrm{MLE}}$ for surfaces with highly polar surface sites, and thus indicate that collective aspects of water's interfacial polarization can be accounted for by specifying the average electrostatic state of the local environment.
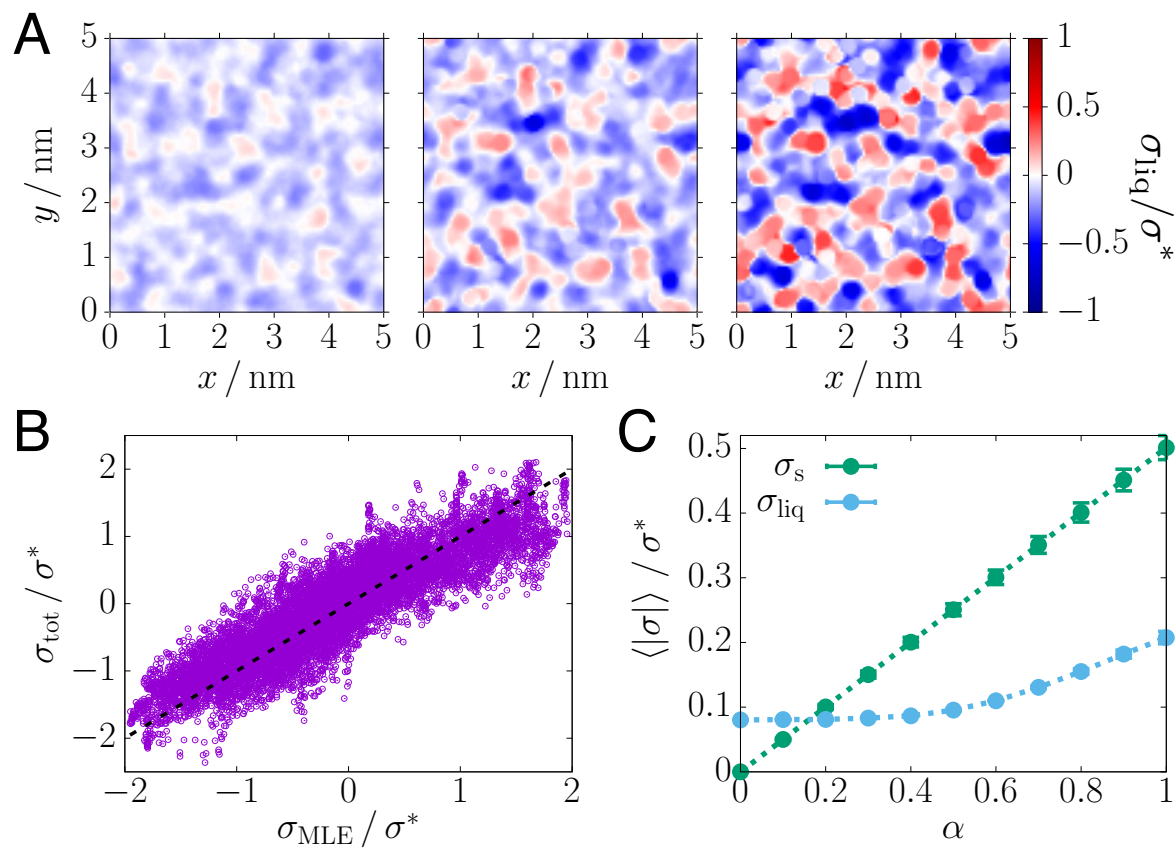

Fig. 3. Role of water-water interactions in the response of water's interfacial molecular structure to surface charge. (A) Spatial maps of $\sigma_{\text {liq }}$ for the surface analyzed in Fig. 2 , with $\alpha=0.4$ (left), 0.7 (center), and 1 (right). The shading indicates the charge density value in units of $\sigma^{*}$ as given in the color bar on the right. (B) Scatter plot between $\sigma_{\text {tot }}$ and $\sigma_{\mathrm{MLE}}$ for $\alpha=1.0$. The dashed line represents the exact correspondence, $\sigma_{\text {tot }}=\sigma_{\mathrm{MLE}}$. (C) Plots of the $\alpha$ dependence of $\left\langle\left|\sigma_{\mathrm{s}}\right|\right\rangle$ and $\left\langle\left|\sigma_{\text {liq }}\right|\right\rangle$, shown in green and sky-blue circles, respectively. The dotted lines are a guide to the eye.

We can gain insight into the role of surrounding waters in determining local polarization response by considering the relative contributions of $\sigma_{\mathrm{s}}$ and $\sigma_{\text {liq }}$ to $\sigma_{\text {tot }}$. Figure $3 \mathrm{C}$ contains a plot of the average (over all surface points) magnitude of the perpendicular electric field arising from the surface itself and the surrounding liquid as a function of $\alpha$. We report these quantities in terms of their equivalent surface charges, $\sigma_{\mathrm{s}}$ and $\sigma_{\text {liq. }}$. We observe a roughly linear increase in the contribution of the surface with $\alpha$, as expected. However, we find a more complicated trend arising from the surrounding liquid, whereby $\sigma_{\text {liq }}$ exhibits little change for $0 \leq \alpha \lesssim 0.4$ and a sharp increase for $\alpha \gtrsim 0.4$. This increase reflects the growing importance of surface-bound water molecules in influencing the polarization response of their surroundings. Due to this influence, the polarization response of a water interface cannot be reliably predicted based on the surface charge alone, except for cases where surface polarity is sufficiently uniform or sufficiently small. 
Dynamic local polarization at the protein-water interface. We extend our approach to study protein surfaces, which are both chemically and topographically heterogeneous as well as dynamic due to the thermal fluctuations of the backbone and the side chains. By including the influence of equilibrium protein fluctuations, we can assess the role of conformational dynamics and intra-protein interactions, such as salt bridges, in determining the polarization response of the surrounding aqueous environment. We illustrate this role in the context of the Escherichia coli chemotaxis signaling protein, CheY (PDB code: 1JBE), which we take as a representative model protein system. The backbone structure of this 128 residue protein, as illustrated in Fig. 4A, presents 90-100 surface residues in the folded state, 33 of which have charged side chains capable of forming salt bridges.

We analyze the aqueous interfacial polarization response by computing $\sigma_{\mathrm{s}}$ and $\sigma_{\mathrm{MLE}}$ on an array of points along the protein surface (see SI Appendix for computational details). Figure 4B illustrates how these computed values relate when the protein structure is fixed in a single folded configuration. We observe that the qualitative features of the surface charge distribution, such as the location and shape of positively and negatively charged regions, are captured in $\sigma_{\mathrm{MLE}}$. This observation implies that water's local polarization response contains information about the underlying protein surface charge. However, the point-by-point agreement between $\sigma_{\mathrm{s}}$ and $\sigma_{\mathrm{MLE}}$ is not quantitative, with a MUE of 0.46 , indicating a prevalence of non-local correlations in water's interfacial polarization response.

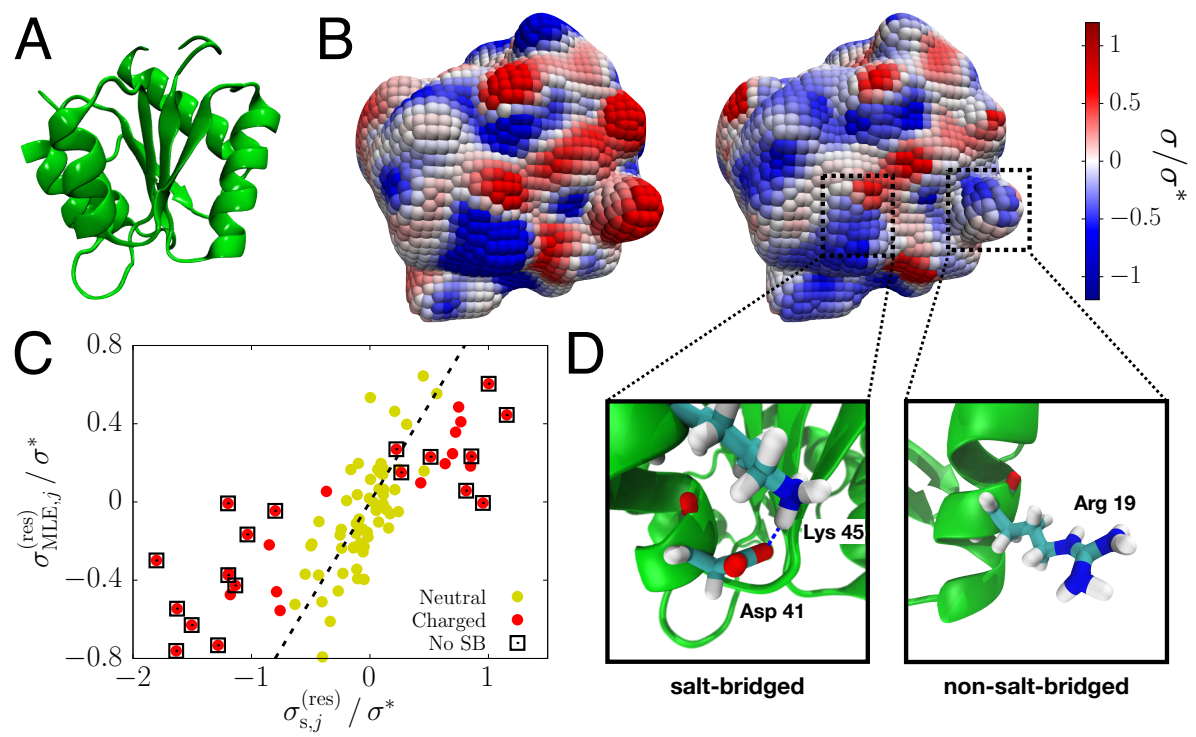

Fig. 4. Water's interfacial polarization response around a protein. (A) Simulation snapshot of the CheY protein (surrounding water molecules and ions are omitted for clarity) showing its structural backbone. (B) Spatial maps of $\sigma_{\mathrm{s}}$ (left) and $\sigma_{\mathrm{MLE}}$ (right) computed for points along the protein surface. (C) Scatter plot between $\sigma_{\mathrm{s}, j}^{(\mathrm{res})}$ and $\sigma_{\mathrm{MLE}, j}^{\text {(res) }}$ for each surface residue. We distinguish residues with neutral side chains and charged side chains by yellow and red points, respectively, and we indicate non-salt-bridged (No $\mathrm{SB}$ ) residues with an additional box around the point. The dashed line corresponds to the perfect correlation, $\sigma_{\mathrm{MLE}, j}^{(\mathrm{res})}=\sigma_{\mathrm{s}, j}^{\text {(res) }}$. (D) An illustration of the differences in side chain alignment for salt-bridged and non-salt-bridged residues.

To gain physical insight, we compute the net polarization response of water around each surface residue. Specifically, we identify the nearest residue of each surface point and then average $\sigma_{\mathrm{MLE}}$ over all points belonging to each individual residue. So the apparent surface charge density of residue $j$ is defined as,

$$
\sigma_{\mathrm{MLE}, j}^{(\mathrm{res})}=\frac{1}{N_{\mathrm{surf}}(j)} \sum_{i \in n(j)}^{N_{\mathrm{surf}}(j)} \sigma_{\mathrm{MLE}, i},
$$


where $n(j)$ is the set of surface points whose nearest residue is $j$ and $N_{\text {surf }}(j)$ is the number of these points. We define $\sigma_{\mathrm{s}, j}^{(\mathrm{res})}$ in an analogous way. Figure $4 \mathrm{C}$ contains a scatter plot of these calculations, illustrating that the correspondence between interfacial polarization and local surface charge differs significantly between neutral and charged residues. Specifically, by comparing the slopes of the linear best fit to each set of points, it appears that $\sigma_{\mathrm{MLE}}$ is systematically less sensitive to $\sigma_{\mathrm{s}}$ around charged residues $($ slope $=0.34$ ) than neutral residues (slope $=0.87$ ). Notably, this relative insensitivity was not observed for the model surface (Fig. 2).

We observe that when charged residues are involved in the formation of salt bridges, they tend to exhibit smaller differences between $\sigma_{\mathrm{MLE}, j}^{(\mathrm{res})}$ and $\sigma_{\mathrm{s}, j}^{(\mathrm{res})}$. We hypothesize that the origin of these smaller differences is related to the geometric arrangements of the side chains which can affect the lateral correlations of nearby interfacial water molecules. The side chains of typical charged residues can protrude into the surrounding water interface, however, the formation of a salt bridge tends to flatten side chains' orientations so that they align parallel to the local protein surface. We illustrate this orientational difference in Fig. 4D. While flattened side chains present a distinct set of charged groups on which interfacial water molecules can be pinned, those that protrude into the surrounding liquid contribute to disrupting local water structure, which is far from the liquid interface, perhaps in the second or third hydration shell. Consequently, the aqueous interfacial polarization near salt-bridged side chains is enhanced whereas the local hydration environment near non-salt-bridged ones is depolarized in a way that extends beyond simple polarization.

The data presented in Fig. 4 was generated for a single fixed configuration of the protein. However, the local hydration structure of a protein can be quite sensitive to equilibrium protein conformational fluctuations. We examine the influence of protein dynamics by computing time-resolved values of $\sigma_{\mathrm{MLE}, j}^{(\mathrm{res})}$ and $\sigma_{\mathrm{s}, j}^{(\mathrm{res})}$. Specifically, we define $\sigma_{\mathrm{MLE}, j}^{(\mathrm{res})}(t)$ and $\sigma_{\mathrm{s}, j}^{(\mathrm{res})}(t)$ by analyzing water configurations only within a 20-ps time window from time $t$ (see SI Appendix for computational details). Figure 5A contains a plot of $\Delta \sigma_{j}^{(\mathrm{res})}(t)=\sigma_{\mathrm{MLE}, j}^{(\mathrm{res})}(t)-\sigma_{\mathrm{s}, j}^{(\mathrm{res})}(t)$ for two specific residues over $10 \mathrm{~ns}$ of the simulated protein dynamics with no restraint on the conformation. We find that the apparent surface charge surrounding the neutral residue, Asn62, does not exhibit a significant difference from the actual surface charge as $\Delta \sigma_{\text {Asn62 }}^{\text {(res) }}$ fluctuates steadily around zero. We observe similar behavior for other neutral surface residues. On the other hand, the time series for $\Delta \sigma_{j}^{(\text {res })}$ for the charged residue, Glu67, exhibits large fluctuations that are indicative of two distinct underlying charge states, one at $\Delta \sigma_{\mathrm{Glu} 67}^{(\mathrm{res})} \approx 1$ and the other at $\Delta \sigma_{\mathrm{Glu} 67}^{(\mathrm{res})} \approx 1.5$. We attribute these dynamics to the making and breaking of a salt bridge. We support this claim by comparing the fluctuations of $\Delta \sigma_{\mathrm{Glu} 67}^{(\mathrm{res})}$ to the fluctuations in the distance between Glu67 and Lys70, $d_{\mathrm{SB}}(67,70)$, which act as an intermittent salt bridge pair. We observe that the large changes in $\Delta \sigma_{\mathrm{Glu} 67}^{\text {(res }}$ coincide with those of $d_{\mathrm{SB}}(67,70)$, indicating that salt bridge formation leads to a concomitant increase in the local solvent polarization. We provide more direct evidences for the relationship between salt bridge formation and local solvent polarization by analyzing $\sigma_{\text {Glu67 }}^{(\text {res })}(t)$ in SI Appendix, Figs. S7A-S7B.

We further highlight the distinction between neutral, charged, and salt-bridged residues by computing the probability distribution of $\Delta \sigma_{j}^{(\text {res })}$ for each residue population. As illustrated in Fig. 5B, the distribution for neutral residues is centered around zero, with a relatively narrow distribution (variance $=0.055$ ). In contrast, the distribution of $\Delta \sigma_{j}^{\text {(res) }}$ for negatively charged residues is relative broad (variance $=0.42)$ and centered at a positive value of $\Delta \sigma^{(\mathrm{res})}$. This non-zero positive mean implies that the hydration layer around negatively charged residues tends to be less polarized 

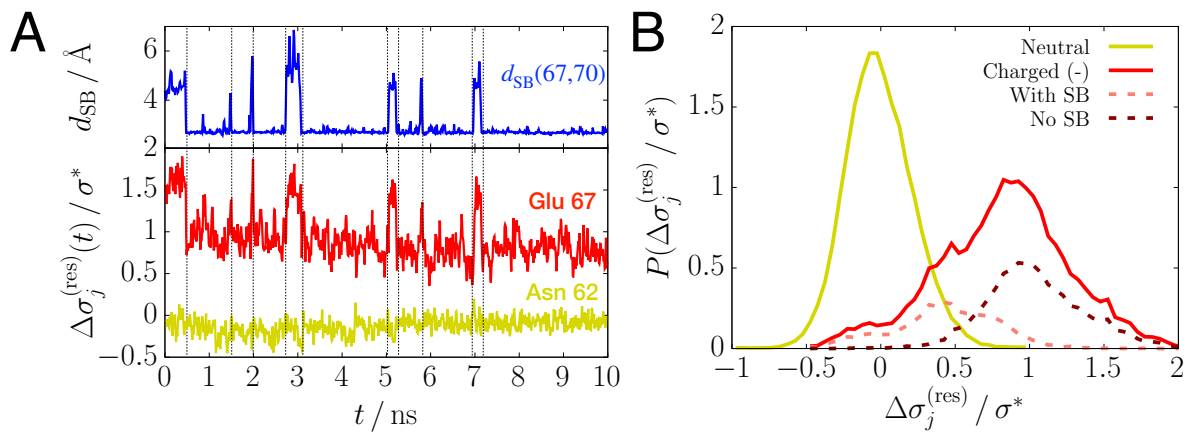

Fig. 5. Water's interfacial molecular structure reveals the dynamic context of nonlocal interactions between the charged residues. (A) Plots of $\Delta \sigma_{j}^{\text {(res) }}(t)$ for two specific residues computed from a 10-ns trajectory of the dynamic protein (red and yellow lines, bottom), where the fluctuations of $\Delta \sigma_{\text {Glu67 }}^{\text {(res) }}(t)$ are coupled with the salt bridge formation

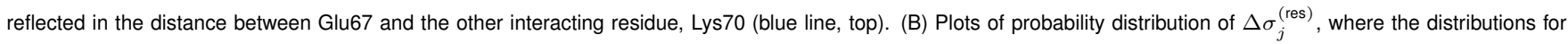
residues with net charge of zero and negative net charge are shown in yellow and red solid lines, respectively. For the negatively charged residues, the distribution is further decomposed into the cases with and without salt bridges (SB), which are shown in light-red and dark-red dashed lines, respectively.

than one would expect based on the residue charge alone. We find an analogous phenomena for positively charged residues, i.e., giving rise to a broad distribution centered at negative values of $\Delta \sigma^{\text {(res) }}$ (SI Appendix, Fig. S7C). Figure 5B also contains distributions for the subpopulations of salt-bridged and non-salt-bridged negatively charged residues. Analysis of these subpopulations highlights that salt bridge formation has the effect of systematically polarizing the local hydration environment. Overall, the results from the dynamic protein further rationalize the implications from the static case.

Qualitative differences in the polarization response at the model and protein surfaces. We now remark on notable similarities and differences between the results generated for our tunable model surface and those generated for the CheY protein. We only find close correspondence between $\sigma_{\mathrm{MLE}}$ and $\sigma_{\mathrm{s}}$ in the case where the magnitude of local surface charge is low, such as when $\alpha \leq 0.4$ or in the vicinity of neutral surface residues. Under these low charge conditions, i.e., having local Coulomb interactions below approximately $0.59 \mathrm{kcal} / \mathrm{mol}$ or $1 k_{B} T$, the spatial characteristics of water's interfacial polarization response will mirror that of the underlying surfaces. However, when surfaces have charge sites that are strongly attractive to water molecules, the spatial characteristics of the interfacial polarization response are influenced by lateral correlations. These correlations can be accounted for by resolving the electric field contributions of the surrounding liquid, but also are affected by the local topography of the surface, including the effects due to the protrusion of charged side chains into the liquid.

Our results highlight the significant influence of protein conformational fluctuations on the polarization response of the surrounding aqueous interface. Along with thermal fluctuations in the specific relative positions of the charged surface residues, the formation of salt bridges has a large local effect on interfacial polarization. Specifically, salt bridge formation contributes to enhancing the effects of the fields that emerge from the side chains that are involved. The transient dynamics of salt bridge formation and deformation are thus relayed to the surrounding liquid via changes in water's interfacial polarization response. These changes may play a role in modulating the water-mediated effects that drive conformational changes (38-40) or the nascent stages of protein-protein (41) or protein-ligand binding (2).

\section{Methods}


Maximum likelihood estimation. We characterize the aqueous interfacial molecular structure of a particular interfacial system by sampling values of $\vec{\kappa}$ and comparing them to $P(\vec{\kappa} \mid$ ref $)$, where the reference system is varied systematically in the specific surface feature. We describe the variation of the reference system using a parameter for the given surface feature, $\sigma$ (e.g., surface charge density for this study), such that the probability for observing a particular orientation in the reference system is also parametrized as $P(\vec{\kappa} \mid \sigma)$. With this parametrization, we can compare quantitatively the sampled molecular orientations, $\mathbb{K}=\left\{\vec{\kappa}_{1}, \vec{\kappa}_{2}, \cdots, \vec{\kappa}_{N}\right\}$, to those from reference interfacial systems for different $\sigma$, based on the likelihood function given by

$$
\mathcal{L}(\sigma \mid \mathbb{K})=\prod_{i=1}^{N} P\left(\vec{\kappa}_{i} \mid \sigma\right)
$$

This quantity represents the probability that the given set of molecular configurations occurs spontaneously in the reference system having the surface property of $\sigma \cdot \mathcal{L}(\sigma \mid \mathbb{K})$ has a larger value when the interfacial molecular structure is closer to that of the reference system with $\sigma$. Therefore, the value of $\sigma$ that maximizes the likelihood function,

$$
\sigma_{\mathrm{MLE}}=\underset{\sigma}{\arg \max } \mathcal{L}(\sigma \mid \mathbb{K})
$$

reflects the optimal extent of the given surface property which underlies water's interfacial molecular structure in the system of interest. We can also quantify the likelihood function with spatial and temporal resolution by analyzing the molecular configurations sampled at a given surface location, $\vec{r}_{\mathrm{s}}$, and time, $t$. Maximizing such a likelihood function will specify $\sigma_{\mathrm{MLE}}\left(\vec{r}_{\mathrm{s}}, t\right)$. More details about computing $\sigma_{\mathrm{MLE}}\left(\vec{r}_{\mathrm{s}}, t\right)$ and its time average are provided in SI Appendix.

Simulation details. To obtain the reference orientational distributions, $P\left(\vec{\kappa} \mid \sigma_{\mathrm{s}}\right)$, we simulated a slab of liquid water in contact with a surface of given charge density in the NVT ensemble using the LAMMPS package (42). 2,474 SPC/E water molecules (43) were put into a simulation cell with dimensions $5 \times 5 \times 12 \mathrm{~nm}^{3}$ measured in $x$-, $y$-, and $z$-directions, respectively. As the simulation cell is long enough in the $z$-dimension, water molecules spontaneously condense into a slab with a thickness of $\sim 3 \mathrm{~nm}$ at $298 \mathrm{~K}$. To mimic a charged surface, point charges were placed on a square lattice with a $1 \AA$ lattice constant at the bottom of the simulation cell along with the 9-3 Lennard-Jones (LJ) potential which represents soft repulsive interactions against water $(44,45)$. The LJ potential between the surface and individual water molecules is given by

$$
u(r)=\epsilon_{\mathrm{LJ}}\left[\frac{2}{15}\left(\frac{\sigma_{\mathrm{LJ}}}{r}\right)^{9}-\left(\frac{\sigma_{\mathrm{LJ}}}{r}\right)^{3}\right]+\frac{13}{15} \epsilon_{\mathrm{LJ}}, r<\sigma_{\mathrm{LJ}}
$$

where $r$ is distance of a water molecule from the surface and the same LJ parameters were used for $\epsilon_{\mathrm{LJ}}$ and $\sigma_{\mathrm{LJ}}$ as those for $\mathrm{SPC} / \mathrm{E}$ water, i.e., $\epsilon_{\mathrm{LJ}}=0.650 \mathrm{~kJ} / \mathrm{mol}$ and $\sigma_{\mathrm{LJ}}=3.166 \AA$. One half of the surface (i.e., $x<25 \AA$ ) has positive charges of $q_{\mathrm{s}}$ and the other half (i.e., $x>25 \AA$ ) has negative charges of $-q_{\mathrm{s}}$, so the entire system is electrically neutral. The molecular orientations were sampled from the regions of $5 \AA<x<20 \AA$ and $30 \AA<x<45 \AA$ to represent the statistics for the surfaces with charge densities, $\sigma_{\mathrm{s}}=q_{\mathrm{s}} / \AA^{2}$, and $\sigma_{\mathrm{s}}=-q_{\mathrm{s}} / \AA^{2}$, respectively. The simulation cell was periodically replicated along $x-y$ directions, and the long-range part of electrostatic interactions were computed by the two-dimensional Particle Mesh Ewald method with a cutoff distance of $10 \AA$ (46). Molecular geometries of water were constrained by the SHAKE algorithm (47). Propagation of dynamics was based on the standard velocity-Verlet integrator with a time step of 2 fs. Water molecules were coupled to the Langevin thermal bath at $T=298 \mathrm{~K}$. The system was equilibrated for 0.1 ns prior to the production run of 2 ns along which a simulation snapshot was taken every 0.1 ps. For a surface of given change density, statistics were collected from six independent trajectories.

ACKNOWLEDGMENTS. We acknowledge useful discussions with Professors Songi Han, Liang Shi, and Rick Remsing. 
This work was supported by the National Science Foundation under CHE-1654415 and also partially (SS) by the Kwanjeong Educational Foundation in Korea.

1. Ball $P$ (2008) Water as an active constituent in cell biology. Chemical Reviews 108(1):74-108

2. Chung E, et al. (1998) Mass spectrometric and thermodynamic studies reveal the role of water molecules in complexes formed between SH2 domains and tyrosyl phosphopeptides. Structure 6(9):1141-1151.

3. Papoian GA, Ulander J, Wolynes PG (2003) Role of water mediated interactions in protein-protein recognition landscapes. Journal of the American Chemical Society 125(30):9170-9178.

4. Fernández A, Scheraga HA (2003) Insufficiently dehydrated hydrogen bonds as determinants of protein interactions. Proceedings of the National Academy of Sciences of the United States of America 100(1):113-118.

5. Levy Y, Onuchic JN (2006) Water Mediation in Protein Folding and Molecular Recognition. Annual Review of Biophysics and Biomolecular Structure 35(1):389-415.

6. Beuming T, et al. (2012) Thermodynamic analysis of water molecules at the surface of proteins and applications to binding site prediction and characterization. Proteins: Structure, Function and Bioinformatics 80(3):871-883.

7. Pan AC, et al. (2019) Atomic-level characterization of protein-protein association. Proceedings of the National Academy of Sciences of the United States of America 116(10):4244-4249.

8. Barnes R, et al. (2017) Spatially Heterogeneous Surface Water Diffusivity around Structured Protein Surfaces at Equilibrium. Journal of the American Chemical Society 139(49):17890-17901.

9. Shin S, Willard AP (2018) Characterizing Hydration Properties Based on the Orientational Structure of Interfacial Water Molecules. Journal of Chemical Theory and Computation 14(2):461-465.

10. Weiß RG, Heyden M, Dzubiella J (2015) Curvature Dependence of Hydrophobic Hydration Dynamics. Physical Review Letters 114(18):19-21.

11. Rego NB, Xi E, Patel AJ (2019) Protein Hydration Waters Are Susceptible to Unfavorable Perturbations. Journal of the American Chemical Society 141(5):2080-2086.

12. Heyden M (2019) Heterogeneity of water structure and dynamics at the protein-water interface. Journal of Chemical Physics 150(9):094701.

13. Shultz MJ, Schnitzer C, Simonelli D, Baldelli S (2000) Sum frequency generation spectroscopy of the aqueous interface: Ionic and soluble molecular solutions. International Reviews in Physical Chemistry 19(1):123-153.

14. Richmond GL (2002) Molecular bonding and interactions at aqueous surfaces as probed by vibrational sum frequency spectroscopy. Chemical Reviews 102(8):2693-2724.

15. Shen YR, Ostroverkhov V (2006) Sum-Frequency Vibrational Spectroscopy on Water Interfaces: Polar Orientation of Water Molecules at Interfaces. Chemical Reviews 106(4):1140-1154.

16. Ebbinghaus S, et al. (2007) An extended dynamical hydration shell around proteins. Proceedings of the National Academy of Sciences of the United States of America 104(52):20749-20752.

17. Heyden M, et al. (2008) Long-range influence of carbohydrates on the solvation dynamics of water-answers from terahertz absorption measurements and molecular modeling simulations. Journal of the American Chemical Society 130(17):5773-5779.

18. McCarney ER, Armstrong BD, Kausik R, Han S (2008) Dynamic nuclear polarization enhanced nuclear magnetic resonance and electron spin resonance studies of hydration and local water dynamics in micelle and vesicle assemblies. Langmuir 24(18):10062-10072.

19. Armstrong BD, Han S (2009) Overhauser dynamic nuclear polarization to study local water dynamics. J. Am. Chem. Soc. 131(13):4641-4647.

20. Heugen U, et al. (2006) Solute-induced retardation of water dynamics probed directly by terahertz spectroscopy. Proceedings of the National Academy of Sciences 103(33):12301-12306.

21. Armstrong BD, et al. (2011) Site-specific hydration dynamics in the nonpolar core of a molten globule by dynamic nuclear polarization of water. Journal of the American Chemical Society 133(15):5987-5995.

22. Wen YC, et al. (2016) Unveiling Microscopic Structures of Charged Water Interfaces by Surface-Specific Vibrational Spectroscopy. Physical Review Letters 116(1):016101.

23. Giovambattista N, Debenedetti PG, Rossky PJ (2007) Effect of surface polarity on water contact angle and interfacial hydration structure. Journal of Physical Chemistry B 111(32):9581-9587.

24. Conti Nibali V, Havenith M (2014) New insights into the role of water in biological function: Terahertz absorption spectroscopy and molecular dynamics simulations studies of the solvation dynamics of biomolecules. J. Am. Chem. Soc. 136:12800-12807.

25. Heyden M, et al. (2010) Dissecting the THz spectrum of liquid water from first principles via correlations in time and space. Proceedings of the National Academy of Sciences of the United States of America 107(27):12068-12073.

26. Gaigeot MP, Sprik M, Sulpizi M (2012) Oxide/water interfaces: how the surface chemistry modifies interfacial water properties. Journal of Physics: Condensed Matter 24(12):124106.

27. Bonthuis DJ, Netz RR (2013) Beyond the continuum: How molecular solvent structure affects electrostatics and hydrodynamics at solid-electrolyte interfaces. Journal of Physical Chemistry $B$ 117(39):11397-11413.

28. Remsing RC, Weeks JD (2016) Role of Local Response in Ion Solvation: Born Theory and beyond. Journal of Physical Chemistry B 120(26):6238-6249.

29. Demille RC, Molinero V (2009) Coarse-grained ions without charges: Reproducing the solvation structure of NaCl in water using short-ranged potentials. Journal of Chemical Physics 131(3).

30. Wu Z, Cui Q, Yethiraj A (2010) A new coarse-grained model for water: The importance of electrostatic interactions. Journal of Physical Chemistry B 114(32):10524-10529.

31. Shin S, Willard AP (2018) Water's Interfacial Hydrogen Bonding Structure Reveals the Effective Strength of Surface-Water Interactions. The Journal of Physical Chemistry B 122(26):6781-6789.

32. Ösapay K, Young WS, Bashford D, Brooks CL, Case DA (1996) Dielectric continuum models for hydration effects on peptide conformational transitions. Journal of Physical Chemistry 100(7):26982705.

33. Tamagawa H, Sakurai M, Inoue Y, Ariga K, Kunitake T (1997) Theoretical study of intermolecular interaction at the lipid-water interface. 2. Analysis based on the Poisson-Boltzmann equation. Journal of Physical Chemistry B 101(24):4817-4825.

34. Koehl P, Orland H, Delarue M (2009) Beyond the Poisson-Boltzmann model: Modeling biomolecule-water and water-water interactions. Physical Review Letters 102(8):1-4.

35. Willard AP, Chandler D (2010) Instantaneous liquid interfaces. The Journal of Physical Chemistry B 114(5):1954-1958.

36. Shin S, Willard AP (2018) Three-Body Hydrogen Bond Defects Contribute Significantly to the Dielectric Properties of the Liquid Water-Vapor Interface. Journal of Physical Chemistry Letters 9(7):1649-1654.

37. Shiraga K, Ogawa Y, Kondo N (2016) Hydrogen Bond Network of Water around Protein Investigated with Terahertz and Infrared Spectroscopy. Biophysical Journal 111(12):2629-2641.

38. Bowman GR, Geissler PL (2012) Equilibrium fluctuations of a single folded protein reveal a multitude of potential cryptic allosteric sites. Proceedings of the National Academy of Sciences of the United States of America 109(29):11681-11686.

39. Pezzella M, et al. (2020) Water dynamics around proteins: T- A nd r-states of hemoglobin and melittin. Journal of Physical Chemistry B 124(30):6540-6554. 
40. Regmi R, et al. (2020) Phosphorylation-Dependent Conformations of the Disordered Carboxyl-Terminus Domain in the Epidermal Growth Factor Receptor. Journal of Physical Chemistry Letters 11(23):10037-10044

41. Reichmann D, Phillip Y, Carmi A, Schreiber G (2008) On the contribution of water-mediated interactions to protein-complex stability. Biochemistry 47(3):1051-1060

42. Plimpton S (1995) Fast Parallel Algorithms for Short-Range Molecular Dynamics. Journal of Computational Physics 117:1-19.

43. Berendsen HJC, Grigera JR, Straatsma TP (1987) The Missing Term in Effective Pair Potentials. Journal of Physical Chemistry 91(24):6269-6271.

44. Abraham FF, Singh Y (1977) The structure of a hard-sphere fluid in contact with a soft repulsive wall. The Journal of Chemical Physics 67(5):2384.

45. Lee C, McCammon JA, Rossky PJ (1984) The structure of liquid water at an extended hydrophobic surface. The Journal of Chemical Physics 80(9):4448-4455.

46. Yeh Ic, Berkowitz ML (1999) Ewald summation for systems with slab geometry. Chemical Physics 111(7):3155.

47. Ryckaert JP, Ciccotti G, Berendsen HJ (1977) Numerical integration of the cartesian equations of motion of a system with constraints: molecular dynamics of n-alkanes. Journal of Computational Physics 23(3):327-341. 